\title{
TTR
}

Traduction, terminologie, re?daction

\section{L’interdit et l'inter-dit : la traduisibilité et le sacré}

\section{Alexis Nouss}

Volume 2, numéro 1, 1er semestre 1989

Carrefours de la traduction

URI : https://id.erudit.org/iderudit/037034ar

DOI : https://doi.org/10.7202/037034ar

Aller au sommaire du numéro

Éditeur(s)

Association canadienne de traductologie

ISSN

0835-8443 (imprimé)

1708-2188 (numérique)

Découvrir la revue

Citer cet article

Nouss, A. (1989). L'interdit et l'inter-dit : la traduisibilité et le sacré. TTR, 2(1),

75-88. https://doi.org/10.7202/037034ar d'utilisation que vous pouvez consulter en ligne.

https://apropos.erudit.org/fr/usagers/politique-dutilisation/ 


\title{
L'interdit et l'inter-dit: la traduisibilité et le sacré
}

\begin{abstract}
Alexis Nouss
La Torah a été donnée au Mont Sinai en 70 langues.
\end{abstract}

(Midrach)

Dans le dessin, ferme ou hésitant, de ses territoires, le discours de la traduction rencontre souvent le discours religieux. Cette convergence peut s'expliquer par un intérêt commun pour les dynamiques, contradictoires ou dialectiques, du mystère et de la révélation. Dans le vécu religieux comme dans l'acte de traduire, il semble qu'il y ait à la fois l'affirmation d'un contenu clos, caché, insaisissable, et le désir de connaitre et faire connaître ce contenu. La révélation (texte spirituel ou traduction), au demeurant, n'infirme pas le mystère: un livre révélé n'en épuise pas pour autant son sens de même qu'une traduction n'est jamais définitive.

Pour certains auteurs, le discours de la traduction prend même sa source dans le discours religieux. Les premières traductions naissent de livres religieux à traduire et la volonté de traduire est inhérente au prosélytisme du fait religieux. La Bible, en Occident, a ainsi pu jouer un rôle majeur en tant que creuset de langues et matrice de traductions. La King James Version ou la traduction de Luther le montrent aisément. Par ce même principe, la Bible devint naturellement source primordiale d'inspiration littéraire, poésie première.

Antoine Berman, concluant son ouvrage majeur l'Épreuve de l'Étranger sur les enjeux et difficultés de l'émergence de la traductologie, évoque les résistances rencontrées par la traduction en Occident et énonce ce propos à nos yeux déconcertant:

Elles semblent être originairement d'ordre religieux et culturel. $\grave{A}$ un premier niveau, elles s'ordonnent autour de l'intraduisible comme valeur. L'essentiel d'un texte n'est pas traduisible ou, à supposer qu'il le soit, 
il ne doit pas être traduit. Dans le cas de la Bible, c'est la tradition juive qui représente cette position extrême. Tout comme la «Loi» ne doit pas être «traduite» de l'oral à l'écrit, le texte sacré ne doit pas être traduit dans d'autres langues, sous peine de perdre son caractère «sacré». Ce double refus indique en creux le lien essentiel de l'écrit et de la traduction, pour mieux questionner les deux. (1984, p. 298)

Antoine Berman établit avec raison le parallèle, quant au texte biblique, entre passage de l'oral à l'écrit et passage de la langue mère, l'hébreu, à d'autres langues. Le mouvement est le même que celui du traduire. Mais c'est précisément parce qu'il est semblable que la traduction, dans la tradition juive - notamment talmudique - est non seulement permise mais encouragée. C'est ce que nous voudrions montrer à partir de la littérature talmudique et midrachique ${ }^{1}$, c'est-àdire à partir du lieu où le judaïsme s'est pensé comme différent de la pensée paienne puis grecque, lieu où la croyance s'est articulée en un discours, discours différenciateur dont le mouvement est précisément celui de la translation, de la constante translation. D'où, par essence, contrairement à l'assertion d'Antoine Berman, une attitude favorable à l'acte du traduire.

\section{Targoum et Torah}

Si le mot Targoum (de la racine tirgem qui signifie, notons-le, à la fois expliquer et comprendre) veut dire «traduction» dans l'acception générale, il est utilisé dans la littérature rabbinique pour désigner presque exclusivement la traduction de la Bible en araméen, accrochant ainsi le phénomène du traduire au texte biblique - comme l'une de ses dimensions, l'un de ses déploiements. D'autre part, l'une de ces traductions, le Targoum Onkelos, du nom de son rédacteur, acquit une telle importance à la période talmudique que le rituel l'intégra dans la lecture hebdomadaire de la section du Pentateuque ${ }^{2}$, usage encore courant dans certains milieux orthodoxes.

Dans le judaïsme, la dynamique de la révélation implique la constante translation, que ce soit dans la révélation même, la transmission ou l'interprétation. Le terme même d'hébreu, ivrit en hébreu, évoque le concept de passage.

1. Le Talmud est la transcription écrite des enseignements oraux des maîtres rabbiniques de Palestine et de Babylonie, recueillis du $\mathrm{II}^{\mathrm{e}}$ siècle avant l'ère chrétienne au $\mathrm{VI}^{\mathrm{e}}$ siècle, relatifs à la conduite, au droit, à la morale, au rituel, à la vie sociale et économique. Il comprend deux parties, la Michna et la Guemara. On en connaît deux versions, le Talmud de Babylone et le Talmud de Jérusalem. Le Midrach, quoiqu'intimement lié au Talmud, rassemble des textes à valeur non pratique et non prescriptive, de portée théologique et philosophique.

2. Ainsi qu'en témoigne le Talmud, Traité Bérakhot, 8a. Les références talmudiques de cet article renvoient aux paginations traditionnelles. Les traductions sont de l'auteur. 
Précisons d'emblée que Torah, en hébreu, signifie enseignement et non Loi, ainsi qu'on le comprend habituellement. Ce dernier terme a des connotations de contrainte et d'intouchabilité, d'immuabilité (d'où les fausses idées d'intraduisibilité) alors que le sens véritable, enseignement, donne une direction de transmission nécessaire et donc déjà le mouvement du traduire. La Révélation n'est qu'un premier enseignement qui, en tant que tel, ne peut vivre que s'il est constamment dispensé, «reprise, vie, invention et renouvellement, modalité sans laquelle le révélé, c'est-à-dire une pensée authentiquement pensée, n'est pas possible. [...] La leçon de vérité ne tient pas dans la conscience d'un seul homme, elle éclate vers autrui.» (Lévinas, 1982, p. 99)

$\mathrm{Au}$ demeurant, la nature même du texte révélé instaure ce mouvement. Le texte doit non seulement se lire mais se déchiffrer, le sens en est à découvrir, non à découvert. S'il n'est pas interprété, il reste lettre morte et perd ainsi son caractère révélé puisque le divin se donne avant tout comme source de vie. Il est de la nature de la transcendance, explique Lévinas, d'attendre une herméneutique, car elle «ne se montre qu'en se dissimulant» (Ibid., p. 142) ${ }^{3}$. Le commentaire et la traduction seront donc des voies royales pour approcher cette transcendance reposant au creux d'un texte/enseignement qui devient avant tout "Parole créatrice de parole». (Ouaknin, 1986, p. 29) Lire le texte, c'est accepter que le sens en précède toujours la lecture et que celle-ci ne puisse jamais définitivement l'atteindre, sens proprement inépuisable ${ }^{4}$. On ne s'étonnera dès lors pas que cette perspective accorde un statut différent au texte religieux qui, dans la terminologie hébraïque, n'est plus «l'Écriture» ou «les Écritures», comme le remarque Henri Meschonnic:

Le terme même de Mikra, qui désigne le corpus biblique, à la fois étymologiquement et fonctionnellement, signifie lecture, non pas lecture comme nous parlons d'une lecture qui s'oppose à l'écriture. Mikra suppose l'assemblée pendant laquelle on lit ou on lisait le texte en question, et, cette lecture étant à voix haute, la notion conjugue, indissociablement à mon sens, l'oralité et la collectivité dans la lecture. (1985, p. 51)

3. Voir, sur ce point, tout le chapitre «De la lecture juive des Écritures», Lévinas, 1982, p. 125.

4. «Les procédés de lecture que l'on vient de voir à l'œuvre suggèrent d'abord que l'énoncé commenté excède le vouloir-dire d'où il procède, que son pouvoir-dire dépasse son vouloir-dire, qu'il contient plus qu'il ne contient, qu'un surplus de sens, peut-être inépuisable, reste enfermé dans les structures syntaxiques de la phrase, dans ses groupes de mots, dans ses vocables, phonèmes et lettres, dans toute cette matérialité du dire, virtuellement toujours signifiant. L'exégèse viendrait libérer dans ces signes une signifiance ensorcelée qui couve sous les caractères ou qui se love dans toute cette littérature des lettres.» (E. Lévinas, 1982, p. 135). 
De même, la Halakha qui définit les règles et prescriptions du code religieux de la vie juive prend entièrement source dans les versets bibliques qu'elle interprète pour y trouver ses fondements. Le sens du texte n'est pas un: il est gros potentiellement des sens nécessaires à son application. Cet appel à l'interprétation, cette présence nécessaire et constamment dynamique d'une oralité en regard - en lecture de l'écrit prouvent assez que le texte biblique, révélé, ne peut être reçu et compris que dans un processus traductif.

\section{L'interprète}

Le Talmud met en scène un personnage répondant au nom de métourgeman, d'une grande importance dans l'économie rituelle synagogale. Il s'agit d'un traducteur professionnel du texte biblique en araméen. "Interprète», au double sens que nous offre le français, préciserait davantage sa fonction puisque celle-ci comprenait, outre la traduction proprement dite de la section hebdomadaire lue pendant l'office public du Chabbat, l'ajout d'explications et de commentaires, parfois de son cru.

Les communautés juives exilées en Babylonie avaient bien vite adopté l'araméen en tant que langue vernaculaire. Celles demeurées en Palestine n'agirent pas différemment puisque l'araméen fut en fait la lingua franca dans toute cette région du Proche-Orient pendant la période de domination perse, du $\mathrm{V}^{\mathrm{e}}$ au $\mathrm{III}^{\mathrm{e}}$ siècle avant l'ère chrétienne. Cette perméabilité linguistique apparaît au demeurant dans le texte biblique même ainsi qu'en témoignent certains passages des livres d'Ezra et de Daniel rédigés en araméen.

Si une autorité fait remonter à Ezra la pratique d'une traduction orale et publique de la lecture biblique synagogale, la tradition attribue plutôt à un certain Onkelos la rédaction de la version araméenne. Le passage talmudique suivant nous éclaire en outre sur les motivations d'une telle traduction.

Et Rabbi Jérémie - certains disent Rabbi Hiyya b. Abba - dit aussi: Le Targoum du Pentateuque fut l'œuvre d'Onkelos le prosélyte sous l'autorité de Rabbi Eléazar et Rabbi Josué. Le Targoum des Prophètes fut l'œuvre de Jonathan ben Ouziel sous l'autorité de Haggaï, Zacharie et Malachie [eux-mêmes prophètes]. Et la terre d'Israël se mit à trembler sur une surface de quatre cents parasanges carrés, une voix céleste se fit entendre qui disait: Qui a dévoilé Mes secrets aux humains? Jonathan ben Ouziel s'est dressé et a dit: C'est moi qui ai dévoilé Tes secrets aux humains. Il est bien connu que je ne l'ai pas fait pour ma gloire, ni pour celle de la maison de mon père mais que je l'ai fait uniquement pour Ta gloire afin que les discussions n'augmentent pas en Israël [au sujet des allusions prophétiques] ${ }^{5}$.

5. Talmud de Babylone, Traité Méguilah, 8a. 
Le texte talmudique place ainsi l'acte traducteur non seulement sous l'autorité de maîtres rabbiniques mais sous la supervision de figures prophétiques. Plus encore, il manifeste l'intérêt divin pour l'entreprise et en reconnaît la nécessité pour une lecture humaine du texte biblique.

Cette traduction du Pentateuque, connue sous le nom de Targoum Onkelos, devint la version officielle des communautés babyloniennes quoique sa paternité soit demeurée matière à controverse. Le Talmud de Jérusalem évoque en effet une traduction du Pentateuque en grec, due à un certain Akilas sous la même autorité de $\mathbf{R}$. Eléazar et $\mathbf{R}$. Josué. Cette version est historiquement attestée et l'Akilas du Talmud de Jérusalem revêt de nombreux traits attribués à Onkelos dans le Talmud de Babylone. Quoi qu'il en soit, le Targoum Onkelos disparut de Palestine après la destruction du Second Temple et reçut une rédaction définitive en Babylonie au $\mathrm{III}^{\mathrm{e}}$ siècle de notre ère. Traduction littérale ou paraphrastique, incluant des ajouts et des explications, le texte d'Onkelos acquit un statut quasi canonique au long des siècles (un Midrach, le Sifrei, en fait un objet d'étude au même titre que la Bible et la Michna) et il est mentionné dans divers ouvrages posttalmudiques.

Certaines sources talmudiques nous informent avec précision sur la manière dont le métourgeman, à la synagogue, intercalait ses traductions dans la lecture biblique sans indiquer s'il s'appuyait sur un texte écrit. D'autres (Talmud de Jérusalem) vont jusqu'à interdire l'utilisation d'une version écrite tout en l'autorisant à des fins privées ou pédagogiques. Des versions écrites ont cependant dû exister très tôt puisque nous avons trace, au ler siècle de notre ère, d'une traduction écrite en araméen du livre de Job. À la fin du $\mathrm{II}^{\mathfrak{e}}$ siècle, l'usage oral du Targoum cessa avec la disparition de l'araméen comme langue vernaculaire des communautés juives de Palestine. Mais il était déjà couché par écrit et officialisé. Comme nous l'indiquions plus haut, son intégration à la lecture biblique hebdomadaire s'est poursuivie jusqu'à nos jours dans certains milieux et même publiquement dans les communautés yéménites.

Singulier destin que cette traduction devenue le doublet inséparable du texte révélé. Rappelons qu'elle ne vient pas se substituer à l'original, ce qui serait encore affirmer l'intouchabilité de celui-ci mais qu'elle l'accompagne. Plus encore: elle y a été greffée au point de lui être liée organiquement. Comme si le texte biblique, le texte révélé, ne pouvait se donner, ou du moins être reçu, qu'avec le déploiement, la résonance d'une traduction.

$\mathrm{Au}$ demeurant, cette dimension n'est pas extérieure. Elle est intrinsèque à la nature même du texte biblique, à l'enjeu de la 
révélation. Le geste traducteur est concomitant à l'expression du message divin. Un Midrach commentant le décalogue est très pertinent à cet égard:

Rabbi Néhemiah dit: Qu'est ce mot de anokhi [je, premier mot du Décalogue: «Je suis l'Éternel ton Dieu qui..."]? Il s'agit d'un mot égyptien. Une parabole l'expliquera. Le fils d'un roi avait été fait prisonnier et avait passé de longs jours en captivité, au point d'apprendre la langue de ses geôliers. Lorsque le roi fut victorieux de ses ennemis, il ramena son fils et commença à lui parler dans sa langue. Mais le prince ne comprenait pas. Que fit le roi? Il entreprit de s'adresser au prince dans la langue des geôliers. Dieu agit de même avec les enfants d'Israël. Toutes les années que ceux-ci passèrent en Égypte, ils apprirent à parler l'égyptien. Lorsque Dieu les délivra, Il voulut leur donner la Torah mais ils ne pouvaient l'entendre. Dieu dit alors: Je vais donc m'adresser à eux en égyptien ${ }^{6}$.

La parole fondatrice, la parole de reconnaissance - qui reconnaît le peuple hébreu en même temps qu'elle le fait naître - , celle par laquelle la divinité s'identifie, ce premier mot de l'Alliance, le Midrach nous indique qu'il est prononcé en traduction! Parce qu'au-delà d'une réalité historique ou d'une catégorie ontologique - l'exil -, le commentaire définit la traduction comme dimension inhérente au discours religieux. Ajoutons que le titre de la section du Pentateuque contenant le Décalogue est «Yitro» (Jéthro dans la dénomination francisée), du nom du beau-père de Moïse, personnage singulier qui se rallie à l'aventure spirituelle hébraïque alors qu'il est grand-prêtre d'une religion païenne. C'est avouer que le message de la Révélation n'admet aucune exclusive, ethnique ou linguistique, et que, d'emblée, il se place dans une dynamique d'ouverture maximale et donc dans un constant processus traductif.

$\grave{A}$ un niveau encore plus profond, le commentaire mystique nous livre une réflexion kabbalistique selon laquelle l'hébreu n'a pas le statut exclusif de langue sainte. Cette dimension imprègne en fait les soixantedix langues traditionnellement reconnues comme l'ensemble des langues du monde ${ }^{7}$ et la conscience spirituelle consiste précisément à retrouver cette dimension, quelle que soit la langue utilisée. En d'autres termes, dans cette perspective, il n'y a pas de langue sainte, il n'y a qu'une parole sainte.

C'est en ce sens que la littérature talmudique et midrachique présente la révélation sinaiitique:

Rabbi Yohanan a dit: Quelle est la signification du verset: Dieu a donné Sa parole, Ses bonnes nouvelles en grande quantité [Ps. 68, 12]? -

6. Midrach Tanhoumah, "Yitro", 16, édition S. Buber, Israël, s.d.

7. Commentaire de Rabbi Isaïe Horovitz dans son ouvrage Chney Louhot HaBrit cité par Charles Mopsik, 1984, pp. 11-16. 
Chaque parole qui sortit de la bouche du Tout-Puissant se divisait dans les soixante-dix langues. À l'école de Rabbi Ismaël, on enseignait: [ $M a$ parole ne ressemble-t-elle pas au feu, dit Dieu, J au marteau qui fait voler en éclats le rocher? [Jér. 23, 29] - De même que le marteau se divise [produit sur l'enclume] de nombreuses étincelles, de même chaque parole qui sortit de la bouche de Dieu se divisait dans les soixante-dix langues 8 .

Le Talmud (Talmud de Babylone, Traité Sotah, 32a) va également voir cette multiplication linguistique dans l'inscription de la Torah sur des pierres enduites de chaux, inscription à laquelle procéda Josué (Jos. 8, 30-35). Lévinas, commentant ce passage talmudique, forge à cet égard le concept de «traductibilité intégrale» qu'il comprend comme une exigence essentielle du message biblique. (1982, p. 94)

Cette pensée semblerait s'inscrire, parmi les théories de la traduction, dans le courant que George Steiner dénomme «tradition gnostique» ou «mysticisme linguistique»:

La tradition occulte soutient qu'une langue originelle unique ou UrSprache se cache derrière nos querelles actuelles, derrière la cacophonie des jargons hostiles qui a suivi l'écroulement de la ziggourat de Nemrod. Cet idiome adamique ne permettait pas seulement aux hommes de se comprendre entre eux, de communiquer en toute facilité. Il incarnait, plus ou moins, le logos primitif, l'acte de création instantanée par lequel Dieu avait littéralement "parlé le monde». (1978, p. 66)

George Steiner, avec justesse, ne réduit pas ce courant aux écoles mystiques ou théosophiques puisque dans le chapitre en question de son Après Babel, il cite non seulement Maître Eckhart, Paracelse et Jakob Böhme, mais encore Leibniz, Hamann et Humboldt ainsi que Spinoza et Wittgenstein, Walter Benjamin, Kafka et Borgès pour finir par une comparaison des théories universalistes et relativistes du langage aux accents tout à fait contemporains.

Les spéculations tendant à comprendre l'origine du langage par l'affirmation d'un langage pur, d'un langage fondamental, corrompu ou oublié par l'histoire, semblent difficilement résister à la tentation de figer ce langage en une langue unique, précise, langue première, structurée phonétiquement, lexicalement et grammaticalement, dissimulée dans les arcanes de traditions secrètes mais que des esprits initiés ou illuminés pourraient retrouver et réarticuler.

L'hébreu tient le premier rang des langues candidates à ce rôle d'élection dans le paysage culturel européen. Non seulement parce que c'est la langue de la Bible mais parce que la tradition hébraïque a très tôt et constamment nourri une réflexion sur le phénomène linguistique:

8. Talmud de Babylone, Traité Chabbat, 88b. On retrouve le même enseignement dans trois recueils midrachiques. 
«L'herméneutique juive fournit les têtes de chapitre qui orienteront la réflexion occidentale sur l'essence des langues humaines et l'énigme de leur mise en pièces.» (Steiner, 1978, p. 68)

Or - et précisément parce qu'il s'agit d'herméneutique -, il est possible d'établir une distinction fondamentale entre ces théories à la recherche du «pur langage». Celui-ci peut être soit une autre langue, langue privilégiée, archétypale, mais toujours un système linguistique particulier parmi tous les systèmes linguistiques que l'esprit humain a pu et su développer, soit une langue autre, une langue toujours autre, toujours l'autre de toute langue, jamais figée, jamais en repos, une langue qui n'apparaitrait et n'existerait qu'en regard d'une autre. Langue en traduction et non langue de traduction, simultanément la langue de départ pour celle d'arrivée et la langue d'arrivée pour celle de départ. Notion éminemment dynamique, donc, qui s'opposerait à une conception statique des systèmes linguistiques.

Dans son célèbre et quasi canonique texte sur «la Tâche du traducteur", Walter Benjamin ne sacrifie jamais à la croyance idolâtre que la «parenté suprahistorique de toutes les langues» serait autre chose qu'un mouvement, un «mode» ou une «visée» selon ses termes:

Ainsi la traduction a finalement pour but d'exprimer le rapport le plus intime entre des langues. Il est impossible qu'elle puisse révéler ce rapport caché lui-même, qu'elle puisse le restituer; mais elle peut le représenter en l'actualisant dans son germe ou dans son intensité. [...] Mais le rapport auquel nous pensons, ce rapport très intime entre les langues, est celui d'une convergence originale. Elle consiste en ce que les langues ne sont pas étrangères l'une à l'autre, mais, a priori et abstraction faite de toutes relations historiques, sont apparentées l'une à l'autre en ce qu'elles veulent dire. (Benjamin, 1971, p. 264)

Jouant sur une image bien connue des figurations ésotériques, Walter Benjamin indique que et l'original et sa traduction sont des fragments d'un langage qui les dépasse, "comme les fragments d'une même amphore" (p. 271). Seul l'ensemble des débris ou des fragments pourra reconstituer l'objet ou plus exactement la forme de l'objet.

C'est en dernière analyse au niveau du sens, de sa production et de sa recherche, que se situe cette approche. C'est à ce même niveau que se pose la réflexion sur le langage et la traduction qu'il est possible de repérer dans les sources talmudiques et midrachiques. Comme nous l'avons vu, l'Écriture - au sens des "Saintes Écritures» - se dit en hébreu mikra, d'une racine qui signifie «lecture»:

Dans cette perspective, les multiples sens du Texte lui sont immanents et peuvent apparaître, successivement, dans le même lieu et dans le même terme. Ce qui fonde la diversité des sens est alors à chercher, non plus dans le Texte lui-même ou dans son jeu avec d'autres doctrines et d'autres textes, mais dans le degré d'investissement et de perspicacité du lecteur. [...] Dans cette approche, la lettre et la littéralité ne sont 
pas en question. Il faudrait plutôt parler de «translittéralité»: le sens traverse la lettre et ne lui est pas adjoint conflictuellement ou dans son extinction. La lettre est là mais traversée de sens. (Trigano, 1984, pp. 61-62)

\section{L'interprétation}

Le Talmud livre dans un de ses traités une image d'une beauté baroque: «Par sa souplesse, le roseau mérita de devenir l'instrument servant à écrire le Pentateuque, les Prophètes et les Hagiographes.» ${ }^{9}$ Certes, l'image vient illustrer un développement d'ordre éthique sur la nécessité d'un esprit conciliant; mais, comme les sources talmudiques métaphorisent souvent l'homme en livre, il nous est loisible d'appliquer la souplesse du roseau à l'écriture même dont il va créer les lignes et les courbes. Le roseau plie, accueille, s'absente et dans ce retrait, par ce retrait, affirme sa nature.

C'est dans cette réserve-là, cette modestie, que le Talmud voudrait ici comprendre le texte révélé. Souplesse du sens qui ne peut se lire que dans un geste à la fois d'effacement et d'invitation à l'endroit du lecteur. D'autant que, si la calligraphie rituelle choisit le roseau, c'est aussi parce que celui-ci est creux: l'encre s'y écoule de même que le sens ruisselle à travers le texte sans s'y figer. Le tracé des lettres compte autant que les lettres tracées.

Le judaïsme ne connaît pas de lettre. Des lettres, certes, au point d'en avoir élaboré une science numérologique et de multiples supports méditatifs, mais la lettre, au sens d'un système dogmatique intouchable et médiatisé par un texte sémantiquement cimenté, une signification verrouillée, jamais.

Le texte biblique dans la lecture talmudique est flottant, à l'image des lettres gravées sur les Tables de la Loi qui, selon la tradition, transperçaient la pierre de part en part.

L'acte fondateur du judaïsme en tant que spiritualité unificatrice d'un peuple est une translation, le passage à l'écrit d'un corpus d'enseignements transmis oralement jusque-là: la décision de Rabbi Yéhuda de donner une version écrite de ces traditions va donner naissance au Talmud, creuset de la pensée juive post-biblique.

Cette dimension orale, cependant, est entendue par la tradition midrachique comme inhérente à la révélation écrite du texte du Pentateuque, affirmant donc, pour ainsi dire, un état de traduction permanente.

Un verset du Lévitique, en effet, contient un curieux pluriel: «Telles sont les ordonnances, les institutions et les Torot [pluriel de

9. Talmud de Babylone, Traité Taanith, 20a. 
Torah] que l'Éternel fit intervenir entre lui et les enfants d'Israël, au Mont Sinaï, par l'intermédiaire de Moïse.» (Lév., 26, 46). Le Midrach répond à la difficulté en comprenant qu'il s'agit de l'enseignement écrit et de l'enseignement oral qui, tous deux, furent contenus dans la révélation sinaitique ${ }^{10}$. Or cet enseignement oral n'est pas qu'un corpus de prescriptions rigides dont la seule particularité serait d'être transmis oralement. Ainsi que le terme hébreu l'indique, Tora-chebealpé, c'est-à-dire «Torah-sur-la-bouche», cette oralité-là est dynamique. La bouche est le lieu d'une parole vivante, mouvante, en constante énonciation, flot ininterrompu que le silence n'interrompt pas mais qu'il jalonne.

Révélation sinaïtique double: un texte, celui du Pentateuque, mais aussi un enseignement oral, ensemble de commentaires et de prescriptions, dont l'importance est égale. Cet enseignement n'est pas uniquement destiné à éclairer le texte écrit, parfois obscur. Il contient, dit-on, la totalité des commentaires et des décisions ultérieures pensés par ceux qui se pencheront sur le texte.

Par définition axée sur le vivant, la tradition orale n'aurait jamais dû passer à l'écrit. Mais après les difficultés politiques postérieures au règne des Maccabées, après la destruction du Temple par Titus, la dispersion du peuple juif par Hadrien et la proscription de l'étude des Écritures, la crainte grandit de voir la chaîne de la tradition brisée par l'histoire ou dissoute par l'oubli. Rabbi Yéhuda brisa l'interdit et coucha par écrit l'ensemble des discussions ayant émergé dans les écoles talmudiques.

Le texte produit par Rabbi Yéhuda se nomme en hébreu michna qui signifie répétition, et donc un texte absent/présent selon l'observation de Meschonnic (1982, p. 303), indiquant par son appellation même qu'il n'existe qu'en translation puisque cette répétition est le fruit de voix plurielles dans le temps et l'espace - les diverses écoles d'interprétation depuis le premier exil.

Rabbi Yéhuda brisa l'interdit pour faire place à l'inter-dit. Mais était-ce réellement un interdit? Une discussion talmudique, rapportée en deux traités différents (Témourah et Guittin), nous éclaire à cet égard. Au départ, il semble bien que l'écrit doive appartenir exclusivement à l'écrit et l'oral à l'oral:

Rabbi Abba, le fils de Rabbi Hiyya bar Abba, disait au nom de Rabbi Yohanan: Ceux qui consignent par écrit les lois traditionnelles ont le même statut que ceux qui brûlent la Torah et ceux qui reçoivent leur enseignement n'en tirent aucune récompense. Rabbi Yéhuda, le métourgeman [l'interprète] de Resh Lakish expliquait: Il est écrit (Ex. 34, 27): «Consigne par écrit ces paroles» puis "car c'est à la condition [litt.:

10. Cité par Marc-Alain Ouaknin, 1986, p. 13. 
«sur la bouche»] de ces paroles» afin de t'apprendre que les traditions orales, il t'est interdit de les énoncer à partir de l'écrit et ce qui est écrit, il t'est interdit de l'énoncer de mémoire ".

Mais après ces déclarations de principes, la réflexion talmudique va s'orienter vers d'autres conclusions sur la base de considérations liées au devenir d'une pensée en prise sur le réel. Par la nécessité de constantes réinterprétations de la loi écrite ou par simple crainte de l'oubli des lois orales, les mêmes autorités précédemment invoquées vont permettre le passage de l'écrit à l'oral:

Rabbi Yohanan et Resh Lakish utilisaient le livre de Aggada le jour de Chabbat en s'appuyant sur le verset (Ps. 119, 126): «Le temps est venu d'agir pour Dieu: Ta loi a été renversée» qu'ils expliquaient ainsi: Il vaut mieux enlever une lettre de la Torah plutôt que la Torah tout entière ne soit oubliée en Israël ${ }^{12}$.

Le verset des Psaumes était ainsi lu par ces maîtres de la manière suivante: «Quand le temps est venu d'agir pour l'Éternel, il est possible de modifier la loi."

La discussion telle qu'elle est exposée dans l'autre traité va prendre un cheminement encore plus radical. Après un passage similaire à celui que nous venons de citer, le débat s'engage une page plus loin sur l'importance respective des traditions écrites et orales. Rabbi Eléazar affirme que la majeure partie de la Torah a été transmise par écrit contre l'avis de Rabbi Yohanan qui avance que la transmission orale excède ce que contient le texte écrit. Dans le développement qui suit, la position de Rabbi Yohanan se révèle encore plus ferme. En réponse à Rabbi Yéhuda, l'interprète de Resh Lakish, et à un maître de l'école de Rabbi Ismaël,

Rabbi Yohanan dit: Dieu n'a conclu une alliance avec Israël qu'en considération des traditions transmises oralement, ainsi qu'il est écrit (Ex.34, 27): "Car c'est à la condition [litt. "sur la bouche»] de ces paroles que $j$ 'ai conclu une alliance avec toi et Israëls. ${ }^{13}$

Ainsi pour Rabbi Yohanan, au-delà de la raison pratique, la nécessité du passage des codes, l'appel de la traduction répond à une exigence métaphysique sertie au cœur même de la révélation. Non seulement tradition écrite et tradition orale se valent, mais elles en appellent constamment à la traduction vers l'autre code. La perspective talmudique fait de l'homme conscient, récepteur ou lecteur de ces traditions, avant tout et inlassablement un traducteur.

11. Talmud de Babylone, Traité Témourah, 14b.

12. Ibid.

13. Talmud de Babylone, Traité Guittin, $60 \mathrm{~b}$. 


\section{La Bible traduite ou les 72 interprètes}

Le cas de la Bible des Septante s'offre à nous comme exemplaire de notre problématique. Cette traduction est en effet la version qui inséra le texte biblique dans l'espace culturel et réflexif occidental.

On en connaît l'histoire telle que la rapportent la Lettre d'Aristée puis des ajouts ultérieurs. Le roi Ptolémée II fait venir 72 savants de Jérusalem auxquels il commande une traduction en grec du Pentateuque (ou de la Bible hébraïque tout entière) destinée à la Bibliothèque d'Alexandrie. Or les savants ayant travaillé isolément produisirent une version identique en tous points. Il semble désormais que si une traduction officielle du Pentateuque en grec vit effectivement le jour à Alexandrie au début du $\mathrm{III}^{\mathrm{e}}$ siècle avant l'ère chrétienne, l'initiative en revenait à la communauté judéo-alexandrine qui avait besoin d'une version du texte biblique en langue vernaculaire. Cette traduction fut d'ailleurs suivie de celle des autres livres de la Bible hébraïque. La Bible des Septante connut par la suite une immense fortune dans le monde chrétien alors que le judaïsme la tint en grande suspicion.

D'un grand intérêt pour notre propos est la manière dont le Talmud, au traité Méguilah, évoque cette traduction dans le cadre d'une discussion sur la possibilité de "lire" - entendons "traduire" le texte révélé en une autre langue. De ce débat dont nous n'avons pas ici la place de résumer les arguments, retenons que la question essentielle qui bientôt ressort n'est en aucun cas la possibilité de traduire ou non le texte biblique mais plutôt de savoir si le texte peut être traduit en toutes langues ou exclusivement en grec. L'opinion de Rabbi Siméon ben Gamaliel prévaut, qui privilégie le grec:

Et Rabbi Yohanan dit: Quel est l'argument de Rabbi Siméon b. Gamaliel? - Il est écrit (Gen. 9, 27): "Que Dieu embellisse Japhet et qu'il réside dans les tentes de Sem» [Yavan est le fondateur du peuple grec; Sem, son frère, est l'ancêtre d'Abraham (voir Gen. 10, 10 et 11)]. Ainsi les paroles de Japhet doivent résider dans les tentes de Sem. Et pourquoi pas les paroles de Gog et Magog [autres fils de Japhet]? Rabbi Hiyya b. Abba répondit: La raison en est qu'il est écrit: "Que Dieu embellisse [yaft] Japhet», c'est-à-dire que ce qui fait la beauté de Japhet réside dans les tentes de $\mathrm{Sem}^{14}$.

Or, ainsi que l'explique le commentateur Rachi suivi par d'autres exégètes, cette beauté-là, c'est le langage grec, supérieur à toutes les autres langues des descendants de Japhet. Si le grec est choisi, c'est précisément pour ses qualités linguistiques, sa capacité à bien traduire le message biblique. Au cours de la même discussion, Rab et Samuel, autres autorités talmudiques, s'accordent à dire que la langue grecque convient à tous les peuples ${ }^{15}$.

14. Talmud de Babylone, Traité Méguilah, 9b. Nous renvoyons pour une discussion de ce texte, dans une autre perspective, à Emmanuel Lévinas, pp. 331-362.

15. Ibid., 18a. 
La pensée talmudique se place ainsi d'emblée dans une perspective de traduction, reconnaissant d'une part la nécessité du passage au vecteur linguistique le plus efficace possible pour la diffusion du texte révélé et reconnaissant d'autre part au grec cette qualité discursive qui en fera l'outil d'expression privilégié de la conscience occidentale. La réflexion rabbinique inaugure ici le processus intellectuel qui, de cette période à nos jours, s'articule autour de la fameuse polarité AthènesJérusalem.

\section{Pour une métaphysique de la traduction}

Nous voudrions en manière de conclusion faire la remarque suivante. Le $\mathrm{XX}^{\mathrm{e}}$ siècle a vu l'émergence d'une pensée originale se plaçant explicitement dans la lignée de la réflexion juive ouverte il y a près de deux mille ans par les questionnements talmudiques et revendiquant l'apparentement au message à lire dans le texte biblique. Qu'elles se situent dans la mouvance existentialiste (Martin Buber), critique (Walter Benjamin) ou phénoménologique (Emmanuel Lévinas), entre autres exemples, ces œuvres ne manquent pas d'interroger la problématique du langage et de la traduction. D'autant que toujours ouvertes à la dimension historique, ces pensées, désireuses de réparer les figures d'un sujet brisé dans et par la modernité, rencontrent une histoire où la parole totalitaire - politique ou idéologique - n'est qu'une langue mensongère, traduite, qu'il faut interpréter pour dénoncer.

Le Talmud énonce à plusieurs reprises que «la Torah parle le langage des hommes". Voici l'interprétation qu'en donnent Emmanuel Lévinas puis Shmuel Trigano:

Contraction de l'Infini dans l'Écriture à moins que - et il n'y aurait là aucun appauvrissement de l'idée cartésienne, ni de la gloire de Dieu, ni de Sa proximité religieuse - à moins que ce ne soit la dignité prophétique du langage, capable de signifier toujours plus qu'il ne dit, merveille de l'inspiration où l'homme écoute, étonné, ce qu'il énonce, où déjà il lit l'énoncé et l'interprète, où déjà la parole humaine est écriture. (Lévinas, 1982, p. 7)

C'est comme si la Torah que nous lisions n'était pas la Torah-en-soi, et n'était que l'annonciatrice d'une Torah des profondeurs. Les "hommes" dont il s'agit sont dits: "Bnei-Adam», les fils d'Adam et non pas «benot", les filles... Le langage de la masculinité ne dit pas celui de la féminité, du monde autre, qui ne peut s'y dire que sur le mode métaphorique du transfert infini (ce qui est l'essence de la féminité): fond sans fond de l'essence humaine. (Trigano, 1977, p. 321)

Transfert infini ou langage sur-signifiant, le mouvement de lecture auquel nous invitent Lévinas et Trigano n'est pas sans rappeler la «versabilité infinie» qu'Antoine Berman nous a permis de reconnaître comme pensée romantique. À une différence essentielle près: ce mode de lecture nomade, en incessant transit, s'inscrit dans l'horizon d'une 
révélation qui en garantit les excès et vise à une parole où le vrai serait à la mesure de l'humain.

Antoine Berman appelle à une éthique de la traduction dont il définit ainsi la «visée»: «ouvrir au niveau de l'écrit un certain rapport à l'Autre, féconder le Propre par la médiation de l'Étranger». (1984, p. 16) Mais pour cela, et puisqu'Antoine Berman parle d'éthique, il faudrait un logos régulateur de ces rapports. Où le trouver aujourd'hui, en un temps où une conscience le moindrement critique se doit de constater l'agonie de toute raison. La traduction, dit-il, révèle un «autre versant» (p. 20) de l'œuvre. À cet autre versant, nous préférons, à la suite de Lévinas, un "au-delà» du texte qui n'est pas un lieu mais un geste, une trace ou encore une marge. En d'autres termes, la «pulsion traduisante» qu'Antoine Berman lie sommairement à une «visée métaphysique» (p. 23) ne nous semble pas tant désirer une «autre langue» qu'une langue toujours autre, ainsi que nous l'indiquions plus haut.

C'est dans ce dépassement, ce débordement vers ce qui est toujours l'autre - un visage ou une langue - , que nous voyons la possibilité d'une métaphysique de la traduction qui serait une métaphysique du dialogue.

Université Concordia

\section{Références}

Benjamin, Walter (1971). Mythe et violence. Trad. Maurice de Gandillac. Paris, Denoël.

Berman, Antoine (1984). L'Épreuve de l'Étranger. Culture et traduction dans l'Allemagne romantique. Paris, Gallimard.

LÉvINAS, Emmanuel (1982). L'Au-delà du verset. Paris, Minuit. (1984). Israël, le Judaïsme et l'Europe. Paris, Gallimard.

Meschonnic, Henri (1982). Critique du rythme. Lagrasse, éditions Verdier. (1985). Les États de la poétique. Paris, P.U.F.

Mopsik, Charles (1984). «Traduire est-ce trahir?» Les Nouveaux Cahiers, Paris, $\mathrm{n}^{\circ} 78$ (automne).

Ouaknin, Marc-Alain (1986). Le Livre brûlé. Paris, Lieu Commun.

SteInER, George (1978). Après Babel. Trad. Lucienne Lotringer. Paris, AlbinMichel.

Trigano, Shmuel (1977). Le Récit de la disparue. Paris, Gallimard. (1984). La Demeure oubliée. Paris, Lieu Commun. 